\title{
Stability of the replica-symmetric saddle-point in general mean-field spin-glass models
}

\author{
Katharina Janzen and Andreas Engel, * \\ ${ }^{1}$ Institut für Physik, Carl von Ossietzky Universtität, 26111 Oldenburg, Germany
}

(Dated: November 3, 2018)

\begin{abstract}
Within the replica approach to mean-field spin-glasses the transition from ergodic hightemperature behaviour to the glassy low-temperature phase is marked by the instability of the replica-symmetric saddle-point. For general spin-glass models with non-Gaussian field distributions the corresponding Hessian is a $2^{n} \times 2^{n}$ matrix with the number $n$ of replicas tending to zero eventually. We block-diagonalize this Hessian matrix using representation theory of the permutation group and identify the blocks related to the spin-glass susceptibility. Performing the limit $n \rightarrow 0$ within these blocks we derive expressions for the de Almeida-Thouless line of general spin-glass models. Specifying these expressions to the cases of the Sherrington-Kirkpatrick, Viana-Bray, and the Lévy spin glass respectively we obtain results in agreement with previous findings using the cavity approach.

PACS numbers:
\end{abstract}

\section{INTRODUCTION}

Spin glasses are paradigmatic examples for systems with competing interactions [1]. Both their equilibrium and dynamical behavior shows unique characteristics which are absent in systems without frustration. The concepts and techniques introduced in the theoretical description of spin glasses 2] have found interesting and widespread applications in other, at first sight unrelated fields of science such as complex optimization, error-correcting codes, artificial neural networks, and computational complexity [3, 4].

One of the central features of spin glasses is their non-ergodic low temperature phase characterized by slow relaxation and hysteretic response to external magnetic fields. A thorough theoretical understanding of this phase is available only for mean-field systems where the spin-glass phase is composed of a hierarchy of ergodic components. In the parameter plane spanned by temperature and external magnetic field the high-temperature phase is separated from the glassy low-temperature phase by the so-called de Almeida-Thouless (AT) line [1, 5]. The determination of the AT-line is therefore of central importance in the theoretical analysis of spin-glass models.

Two rather different approaches are by now available to calculate the equilibrium properties of mean-field spin glasses. The replica method [6] starts with $n$ replicas of the system under consideration which after the ensemble average over the quenched disorder interact with each other. The free energy can be determined from a saddle-point integral over order parameters. The trademark of the replica method is the mathematically problematic limit $n \rightarrow 0$ to be performed at the end. In this framework the AT-line is determined by the local stability of the replica-symmetric saddle point [5]. In the cavity method [7, 8] one spin is added to a system of $N$ spins and the stochastic stability of the thermodynamic limit $N \rightarrow \infty$ is used to derive self-consistent equations for the order parameters. Here the AT-line may be obtained by investigating the correlations between two spins which must vanish in the thermodynamic limit for a pure state of a mean-field system [2].

Both methods have been implemented for the analysis of the simplest mean-field spin glass, the SherringtonKirkpatrick (SK) model [9]. For this model the ergodic phase is characterized by a single order parameter and a Gaussian distribution of local magnetic fields. The fluctuations around the replica-symmetric saddle-point are described by an $n(n-1) / 2 \times n(n-1) / 2$ matrix. Its eigenvalues have been determined in [5, 10, 11]. The temperature dependence of these eigenvalues shows that the replica-symmetric saddle-point loses its stability at the phase boundary of the ergodic phase. The detailed form of the AT-line was reproduced within the cavity approach [2].

The situation is less clear for more general mean-field spin-glass models which unlike the SK-model are characterized by non-Gaussian distributions of local fields. Models of this type are in particular important in complex optimization [4, 12]. A prototype of this class is the Viana-Bray (VB) [13] model for a diluted spin glass in which each spin interacts with just a few, randomly selected other spins. Here the AT-line was determined numerically in [14], whereas analytical information is available only near the freezing temperature [13]. The replica treatment of diluted spin glasses and optimization problems is more complicated than that of the SK-model and involves already at the replica-symmetric

*Electronic address: janzen@theorie.physik.uni-oldenburg.de, engel@theorie.physik.uni-oldenburg.de 
level an infinite number of order parameters [15, 16]. A general and elegant approach to this more complicated setting was introduced by Monasson [17]. The fluctuations around the replica-symmetric saddle-point are now characterized by an $2^{n} \times 2^{n}$ matrix which has to be diagonalized in order to assess the stability of replica symmetry. Recently it has been shown [18] that this method may also be used to analyze spin-glass models characterized by coupling distributions with diverging moments such as Lévy glasses [19, 20]. This opens up the possibility to determine the AT-line also for such models within the replica method.

In the present paper we investigate the stability of the replica-symmetric saddle-point for spin-glass models with non-Gaussian field distribution. To this end we implement the approach of Monasson for diluted spin glasses and reduce the determination of the free energy per spin to a saddle-point integral over $2^{n}$ order parameters. The Hessian matrix describing the fluctuations around this saddle-point can be block-diagonalized by exploiting the representation theory of the permutation group [21]. We also build on techniques introduced in [22, 23] for the analysis of replica symmetry breaking in one-dimensional spin glasses. We then identify the blocks which are related to the spin-glass susceptibility $\chi_{\mathrm{SG}}$ the divergence of which signals the onset of spin-glass order. Up to this point the analysis is rather general and uses only the replica structure of the fluctuation matrix. The final diagonalization of the relevant blocks can only be performed after the details of the model under consideration are fixed. We consider three representative examples: the SK model which merely serves as test case for our method, the VB model as example for diluted spin glasses, and the Lévy glass as system with a local field distribution exhibiting long tails. In all cases we provide expressions for the AT-line separating the replica-symmetric part of the phase space from the region characterized by replica symmetry breaking.

The paper is organized as follows. In section \we define the central models of interest, recollect the main steps in the replica-symmetric theory for diluted spin glasses and fix the notation. Section III] contains the analysis of the situation without external magnetic field for which the calculations are significantly simpler. Section [V] is devoted to the general case from which the expressions for the complete AT-lines in the models considered result. Finally, in section $\nabla$ we give some conclusion and discuss open problems. Some more technical steps are relegated to the appendices.

\section{BASIC EQUATIONS}

We consider Ising spins $S_{i}= \pm 1, i=1, \ldots, N$ with random, pairwise interactions specified by a symmetric matrix $J_{i j}$ in an external field $h_{\text {ext }}$. The Hamiltonian is of the general form

$$
H\left(\left\{S_{i}\right\}\right)=-\frac{1}{2} \sum_{(i, j)} J_{i j} S_{i} S_{j}-h_{\mathrm{ext}} \sum_{i} S_{i},
$$

where the first sum runs over all pairs of spins. The couplings $J_{i j}=J_{j i}$ are i.i.d. random variables drawn from a model dependent distribution $P_{M}$. We will consider three examples for this distribution in detail, namely

$$
\begin{aligned}
P_{S K}(J) & :=\sqrt{\frac{N}{4 \pi}} \exp \left(-\frac{N J^{2}}{4}\right) \\
P_{V B}(J) & :=\frac{\kappa}{N} p(J)-\left(1-\frac{\kappa}{N}\right) \delta(J) \\
\hat{P}_{\alpha}(q) & :=\int \mathrm{d} J e^{-i J q} P_{\alpha}(J)=\exp \left(-\frac{|q|^{\alpha}}{N}\right) \quad \alpha \in(0,2]
\end{aligned}
$$

corresponding to the Sherrington-Kirkpatrick (SK) model [9], the Viana-Bray (VB) model [13], and the Lévy spin glass [18] respectively. Here $p(J)$ denotes the distribution of the non-zero bonds in the VB model. The distribution $P_{\alpha}$ for the Lévy spin glass is defined via its characteristic function $\hat{P}_{\alpha}$. Note also that the variance of the Gaussian $P_{S K}$ is twice its standard value such that it coincides with the Lévy case in the limit $\alpha \rightarrow 2$.

The SK model is the paradigmatic case of a fully connected spin-glass in which each spin interacts with each other via weak couplings of order $N^{-1 / 2}$. Complementary, the VB model is characteristic for diluted spin glasses in which each spin interacts with only a few other spins via strong $\mathcal{O}(1)$ couplings. The Lévy spin glass interpolates between these two extremes since each spin interacts with each other spin but most of the couplings are very weak whereas $\mathcal{O}(1)$ couplings per spin are strong.

The large connectivity limit of the VB model leads either to SK- or Lévy-like behaviour in the thermodynamic limit, depending on the existence of the second moment of the distribution $p(J)$. If the second moment exists this limit is defined as

$$
\kappa \mapsto N, \quad p(J) \mapsto \sqrt{N} p(\sqrt{N} J),
$$


and leads to an SK-model due to the central limit theorem. On the other hand, if the distribution $p(J)$ has a power law tail: $p(J) \simeq_{|J| \rightarrow \infty}|J|^{-(\alpha+1)}, \quad \alpha \leq 2$, the large connectivity limit is given by:

$$
\kappa \mapsto N, \quad p(J) \mapsto N^{\frac{1}{\alpha}} p\left(N^{\frac{1}{\alpha}} J\right)
$$

The limits coincide for $\alpha=2$ as it should be.

In the framework of the replica approach the free energy density $f$, is expressed through the disorder averaged replicated partition function $\overline{Z^{n}(\beta)}$ via

$$
f:=-\lim _{N \rightarrow \infty} \frac{1}{\beta N} \overline{\ln Z(\beta)}=-\lim _{N \rightarrow \infty} \frac{1}{\beta N} \lim _{n \rightarrow 0} \frac{\overline{Z^{n}(\beta)}-1}{n},
$$

where for integer values of $n$ the replicated partition function is given by

$$
Z^{n}(\beta)=\sum_{\left\{S_{i}^{a}\right\}} \exp \left(-\beta \sum_{a=1}^{n} H\left(\left\{S_{i}^{a}\right\}\right)\right) .
$$

After performing the disorder average in (8) the trace over the spin variables can be transformed into a $2^{n}$-dimensional integral over order parameters [17]

$$
c(\vec{\sigma})=\frac{1}{N} \sum_{i} \delta\left(\vec{S}_{i}, \vec{\sigma}\right),
$$

where $\vec{\sigma}=\left\{\sigma_{a}\right\}_{a=1 \ldots n}$ stands for an Ising spin vector with $n$ components and $\delta(\vec{S}, \vec{\sigma})$ denotes the Kronecker- $\delta$. Hence

$$
\left\langle Z^{n}(\beta)\right\rangle_{J}=\int \prod_{\vec{\sigma}} \mathrm{d} c(\vec{\sigma}) \delta\left(\sum_{\vec{\sigma}} c(\vec{\sigma})-1\right) \exp \left(-N \beta f_{\text {trial }}(\{c(\vec{\sigma})\})\right),
$$

where the $\delta$-function enforces the constraint

$$
\sum_{\vec{\sigma}} c(\vec{\sigma})=1
$$

resulting from (91). The trial free energy $f_{\text {trial }}$ has two contributions $f_{\text {trial }}=f_{S}+f_{E}$ according to

$$
\beta f_{\text {trial }}(\{c(\vec{\sigma})\})=\sum_{\vec{\sigma}} c(\vec{\sigma}) \ln c(\vec{\sigma})-\left[\frac{1}{2} \sum_{\vec{\sigma}, \vec{\tau}} c(\vec{\sigma}) c(\vec{\tau}) \int \mathrm{d} J G_{M}(J) \exp (\beta J \vec{\sigma} \cdot \vec{\tau})+\beta h_{\operatorname{ext}} \sum_{\vec{\sigma}} c(\vec{\sigma})\left(\sum_{a=1}^{n} \sigma_{a}\right)\right] .
$$

The first term is the entropic contribution $f_{S}$ specifying the number of spin configurations realizing a particular set of order parameters $c(\vec{\sigma})$. The second term $f_{E}$ derives from the Hamiltonian (1) and comprises the interaction energy and the energy in the external magnetic field. This latter contribution as well as $f_{S}$ do not depend on the explicit model considered. The interaction part in $f_{E}$ on the other hand depends on the specific form of the coupling distribution $P_{M}$ which is encoded in $G_{M}(J)$. For the three models specified in (2)-(4) one has

$$
G_{S K}(J)=\delta^{\prime \prime}(J) \quad G_{V B}(J)=\kappa(p(J)-\delta(J)) \quad G_{L}(J)=-\int \frac{\mathrm{d} \tilde{J}}{2 \pi}|\tilde{J}|^{\alpha} \exp (i J \tilde{J}),
$$

respectively, where $\delta^{\prime \prime}$ denotes the second derivative of the $\delta$-function. In the case of the Lévy spin glass we work at imaginary temperatures $\beta=-i k$ as long as $n \neq 0$ [18].

With the form (10) the calculation of the free energy $f$ is reduced to a single site problem as is characteristic for mean-field systems. In the thermodynamic limit $N \rightarrow \infty$ the remaining integrals over the order parameters can be evaluated by the saddle-point method. One therefore has to determine the minima $c_{0}$ of $f_{\text {trial }}(\{c(\vec{\sigma})\})$ satisfying

$$
c_{0}(\vec{\sigma})=\mathcal{L}_{n} \exp \left(\sum_{\vec{\tau}} c_{0}(\vec{\tau}) \int \mathrm{d} J G_{M}(J) \exp (\beta J \vec{\sigma} \cdot \vec{\tau})+\beta h_{\operatorname{ext}} \sum_{a=1}^{n} \sigma_{a}\right),
$$

where $\mathcal{L}_{n}$ is a factor accounting for the constraint (11).

The general solution of the saddle-point equation is a complicated problem. To get some guidance one uses as a first step the assumption of replica symmetry (RS) stipulating that $c_{0}$ depends on $\vec{\sigma}$ only through the sum $\sum_{a=1}^{n} \sigma_{a}$ of 
the vector components. These replica-symmetric order parameters are related to the (replica-symmetric) distribution of local fields $\mathcal{P}(h)$ in the spin glass by [17]

$$
c_{0}(\vec{\sigma})=c\left(\sum_{a=1}^{n} \sigma_{a}\right)=\int \mathrm{d} h \mathcal{P}(h) \frac{\exp \left(\beta h \sum_{a=1}^{n} \sigma_{a}\right)}{(2 \cosh (\beta h))^{n}} .
$$

As is well known the replica-symmetric solution fails at low temperatures which on the formal level is due to the instability of the replica-symmetric saddle-point. To assess this stability the temperature dependent eigenvalues of the Hessian $\mathcal{H}$ describing the quadratic fluctuations around the replica-symmetric saddle-point have to be determined. The matrix elements of $\mathcal{H}$ are given by

$$
\mathcal{H}(\vec{\sigma}, \vec{\tau})=\left.\frac{\beta \partial^{2} f_{\text {trial }}}{\partial c(\vec{\sigma}) \partial c(\vec{\tau})}\right|_{R S}=\frac{\delta_{\vec{\sigma}, \vec{\tau}}}{c\left(\sum_{a=1}^{n} \sigma_{a}\right)}-\int \mathrm{d} J G_{M}(J) \exp (\beta J \vec{\sigma} \cdot \vec{\tau}) .
$$

Similarly to $f_{\text {trial }}$ the Hessian splits into an entropic and an energetic contribution, $\mathcal{H}=\mathcal{H}_{S}+\mathcal{H}_{E}$. Note that the external field does not show up explicitly in $\mathcal{H}$. Nevertheless it influences the stability of the replica-symmetric saddle-point due to the dependence of $c_{0}$ on $h_{\text {ext }}$ as specified by (13). Note also that the expression (15) does not yet account for the constraint (11).

The determination of the eigenvalues of $\mathcal{H}$ may be systematically simplified by exploiting the permutation symmetry of the replica-symmetric saddle-point. To this end it is convenient to express the Hessian as a tensor product of $2 \times 2$ matrices using

$$
e^{\beta J \vec{\sigma} \cdot \vec{\tau}}=\prod_{a=1}^{n} e^{\beta J \sigma_{a} \tau_{a}}=\prod_{a=1}^{n}\left\langle\sigma_{a}\left|\left(e^{\beta J} \hat{\sigma}_{0}+e^{-\beta J} \hat{\sigma}_{1}\right)\right| \tau_{a}\right\rangle=\left\langle\vec{\sigma}\left|\bigotimes_{a=1}^{n}\left(e^{\beta J} \hat{\sigma}_{0}+e^{-\beta J} \hat{\sigma}_{1}\right)\right| \vec{\tau}\right\rangle
$$

for the energetic and

$$
\frac{\delta_{\vec{\sigma}, \vec{\tau}}}{c\left(\sum_{a=1}^{n} \sigma_{a}\right)}=\int \frac{\mathrm{d} r \mathrm{~d} r^{\prime}}{2 \pi} \frac{e^{i r r^{\prime}}}{c(r)} \prod_{a=1}^{n} \delta_{\sigma_{a}, \tau_{a}} e^{-i r^{\prime} \sigma_{a}}=\left\langle\vec{\sigma}\left|\int \frac{\mathrm{d} r \mathrm{~d} r^{\prime}}{2 \pi} \frac{e^{i r r^{\prime}}}{c(r)} \bigotimes_{a=1}^{n} \exp \left(-i r^{\prime} \hat{\sigma}_{3}\right)\right| \vec{\tau}\right\rangle
$$

for the entropic part, respectively. Here the vectors $|\vec{\sigma}\rangle$ are defined as $|\vec{\sigma}\rangle=\left|\sigma_{1}, \sigma_{2}, \ldots \sigma_{n}\right\rangle=\bigotimes_{a=1}^{n}\left|\sigma_{a}\right\rangle$ with $\sigma_{a}= \pm 1$ which span the space $V$ of replicated spin configurations. The matrices

$$
\hat{\sigma}_{0}=\left(\begin{array}{ll}
1 & 0 \\
0 & 1
\end{array}\right), \quad \hat{\sigma}_{1}=\left(\begin{array}{ll}
0 & 1 \\
1 & 0
\end{array}\right), \quad \hat{\sigma}_{3}=\left(\begin{array}{rr}
1 & 0 \\
0 & -1
\end{array}\right) .
$$

are the usual Pauli matrices.

\section{ZERO EXTERNAL FIELD}

The discussion of the eigenvalues of the Hessian $\mathcal{H}$ is relatively straightforward for $h_{\text {ext }}=0$ and $T \geq T_{c}$. We therefore first derive expressions for the eigenvalues in this region and afterwards turn to the more involved situation with $h_{\text {ext }} \neq 0$.

For $h_{\text {ext }}=0$ the saddle-point equation (13) has for all $\beta$ the solution

$$
c\left(\sum_{a=1}^{n} \sigma_{a}\right)=\frac{1}{2^{n}}
$$

which, according to (14), corresponds to $P(h)=\delta(h)$. On physical grounds we expect that this paramagnetic solution is stable at sufficiently high temperatures. For constant $c(s)$ the Hessian (15) for the paramagnetic solution reads

$$
\left\langle\vec{\sigma}\left|\mathcal{H}_{P M}\right| \vec{\tau}\right\rangle=\left\langle\vec{\sigma}\left|2^{n} \bigotimes_{a=1}^{n} \hat{\sigma}_{0}-\int \mathrm{d} J G(J) \bigotimes_{a=1}^{n}\left(e^{\beta J} \hat{\sigma}_{0}+e^{-\beta J} \hat{\sigma}_{1}\right)\right| \vec{\tau}\right\rangle
$$

Its eigenvectors can therefore be constructed from those of $\hat{\sigma}_{1}$, namely

$$
|k\rangle=\frac{1}{\sqrt{2}}\left(|+\rangle+(-1)^{k}|-\rangle\right) \quad k \in\{0,1\} .
$$


The eigenvectors of $\mathcal{H}_{P M}$ may hence be written in the form

$$
|\vec{k}\rangle=\left|k_{1}, \ldots, k_{n}\right\rangle=\bigotimes_{a=1}^{n}\left|k_{a}\right\rangle \quad \text { with } \quad\left|k_{a}\right\rangle \in\{|1\rangle,|0\rangle\} .
$$

The $2^{n}$ mutually orthogonal vectors $|\vec{k}\rangle$ form a basis in the space $V$. With the abbreviation $k=\sum_{a} k_{a}$ we find

$$
\bigotimes_{a=1}^{n}\left(e^{\beta J} \hat{\sigma}_{0}+e^{-\beta J} \hat{\sigma}_{1}\right)|\vec{k}\rangle=(2 \cosh (\beta J))^{n-k}(2 \sinh (\beta J))^{k}|\vec{k}\rangle
$$

and therefore the eigenvalue corresponding to $|\vec{k}\rangle$ is given by

$$
\Lambda_{n}^{\vec{k}}=2^{n}\left(1-\int \mathrm{d} J G_{M}(J) \cosh ^{n}(\beta J) \tanh ^{k}(\beta J)\right) .
$$

Not all of these eigenvalues are, however, relevant for the stability of the saddle-point since the constraint (11) needs still to be taken into account. To do so consider small fluctuations $\delta c(\vec{\sigma})$ around the RS saddle-point $c_{0}(\vec{\sigma})=2^{-n}$. With the notations $\delta c(\vec{\sigma})=\langle\delta c \mid \vec{\sigma}\rangle$ and $\delta \hat{c}(\vec{k})=\langle\delta c \mid \vec{k}\rangle$ we find from (10) to second order in $\delta c(\vec{\sigma})$

$$
\begin{aligned}
\overline{Z^{n}(\beta)} & \approx \exp \left(-N \beta f_{\text {trial }}\left(\left\{2^{-n}\right\}\right)\right) \int \prod_{\vec{\sigma}} \mathrm{d} \delta c(\vec{\sigma}) \delta\left(\sum_{\vec{\sigma}} \delta c(\vec{\sigma})\right) \exp \left(-\frac{N}{2} \sum_{\vec{\sigma}, \vec{\tau}} \delta c(\vec{\sigma}) \mathcal{H}_{P M}(\vec{\sigma}, \vec{\tau}) \delta c(\vec{\tau})\right) \\
& =\exp \left(-N \beta f_{\text {trial }}\left(\left\{2^{-n}\right\}\right)\right) \int \prod_{\vec{k}} \mathrm{~d} \delta \hat{c}(\vec{k}) \delta\left(2^{\frac{n}{2}} \delta \hat{c}(\overrightarrow{0})\right) \exp \left(-\frac{N}{2} \sum_{\vec{k}} \Lambda_{\vec{k}} \delta \hat{c}(\vec{k})^{2}\right) \\
& =\exp \left(-N \beta f_{\text {trial }}\left(\left\{2^{-n}\right\}\right)-\frac{n}{2} \ln 2\right) \int \prod_{\vec{k} \neq \overrightarrow{0}} \mathrm{~d} \delta \hat{c}(\vec{k}) \exp \left(-\frac{N}{2} \sum_{\vec{k} \neq \overrightarrow{0}} \Lambda_{\vec{k}} \delta \hat{c}(\vec{k})^{2}\right) .
\end{aligned}
$$

Here we have used the transformations

$$
\sum_{\vec{\sigma}} \delta c(\vec{\sigma})=\sum_{\vec{\sigma}}\langle\delta c \mid \vec{\sigma}\rangle=\sum_{\vec{k}}\langle\delta c \mid \vec{k}\rangle \sum_{\vec{\sigma}}\langle\vec{k} \mid \vec{\sigma}\rangle=\sum_{\vec{k}} \delta \hat{c}(\vec{k}) 2^{\frac{n}{2}} \delta_{\vec{k}, \overrightarrow{0}}=2^{\frac{n}{2}} \delta \hat{c}(\overrightarrow{0})
$$

and

$$
\sum_{\vec{\sigma}, \vec{\tau}} \delta c(\vec{\sigma}) \mathcal{H}(\vec{\sigma}, \vec{\tau}) \delta c(\vec{\tau})=\sum_{\vec{k}} \Lambda_{n}^{\vec{k}} \delta \hat{c}(\vec{k})^{2}
$$

Consequently $|\overrightarrow{0}\rangle$ is perpendicular to the constraint (11) and the integration in the corresponding direction is suppressed by the $\delta$-function in (10). The value of $\Lambda_{n}^{\overrightarrow{0}}$ is therefore not relevant for the convergence of the integral (25).

The limit $n \rightarrow 0$ can be performed now for every eigenvalue $\Lambda_{n}^{(k)}:=\Lambda_{n}^{\vec{k}}$ corresponding to the eigenspace spanned by eigenvectors with magnetization $k$

$$
\Lambda_{P M}^{(k)}=\lim _{n \rightarrow 0} \Lambda_{n}^{\vec{k}}=1-\int \mathrm{d} J G_{M}(J) \tanh ^{k}(\beta J) .
$$

The SG transition is signaled by the divergence of the SG susceptibility given by [1]

$$
\chi_{S G}:=\frac{1}{N} \sum_{i, j} \overline{\left\langle S_{i} S_{j}\right\rangle_{c}^{2}}=\frac{1}{N} \sum_{i, j} \lim _{n \rightarrow 0} \frac{1}{n(n-1)} \sum_{(a, b)}\left\langle S_{i}^{a} S_{j}^{a} S_{i}^{b} S_{j}^{b}\right\rangle_{\mathrm{repl}} .
$$

where the second equality holds only in the paramagnetic phase. The last average is to be taken with respect to all distinct replicas after the disorder average has been performed and yields

$$
\left\langle S_{i}^{a} S_{j}^{a} S_{i}^{b} S_{j}^{b}\right\rangle_{\mathrm{repl}}=\sum_{\left\{S_{i}^{a}\right\}} S_{i}^{a} S_{j}^{a} S_{i}^{b} S_{j}^{b} \exp \left(\frac{1}{2 N} \sum_{i, j} \int \mathrm{d} J G_{M}(J) e^{\beta J \vec{S}_{i} \cdot \vec{S}_{j}}+\beta h_{\mathrm{ext}} \sum_{i=1}^{N} \sum_{a=1}^{n} S_{i}^{a}\right)
$$


Using the same method as for the replicated partition function $\overline{Z^{n}(\beta)}$ it is possible to rewrite (28) as an $2^{n}$-dimensional integral. In the saddle-point approximation one finds using $c_{0}(\vec{\sigma})=2^{-n}$

$$
\begin{aligned}
\chi_{S G} & =N+\lim _{n \rightarrow 0} \frac{N}{n(n-1)} \int \prod_{\vec{\sigma}} \mathrm{d} c(\vec{\sigma}) \delta\left(\sum_{\vec{\sigma}} c(\vec{\sigma})-1\right) \sum_{\vec{\sigma}, \vec{\tau}} c(\vec{\sigma}) c(\vec{\tau})(\vec{\sigma} \cdot \vec{\tau})^{2} \exp \left(-N \beta f_{\text {trial }}(\{c(\vec{\sigma})\})\right) \\
\approx & \lim _{n \rightarrow 0} \frac{N e^{-N \beta f_{\text {trial }}\left(\left\{2^{-n}\right\}\right)}}{n(n-1)} \int \prod_{\vec{\sigma}} \mathrm{d} \delta c(\vec{\sigma}) \delta\left(\sum_{\vec{\sigma}} \delta c(\vec{\sigma})\right) \sum_{\vec{\sigma}, \vec{\tau}} \delta c(\vec{\sigma})(\vec{\sigma} \cdot \vec{\tau})^{2} \delta c(\vec{\tau}) \exp \left(-\frac{N}{2} \sum_{\vec{\sigma}, \vec{\tau}} \delta c(\vec{\sigma}) \mathcal{H}_{P M}(\vec{\sigma}, \vec{\tau}) \delta c(\vec{\tau})\right) \\
& =\frac{1}{\Lambda^{(2)}} .
\end{aligned}
$$

The spin-glass susceptibility is hence directly related to one of the eigenvalues of the Hessian. As expected the divergence of $\chi_{S G}$ at the spin-glass transition corresponds to the instability of the replica-symmetric saddle-point signaled by $\Lambda^{(2)}=0$. We also mention that for non-symmetric coupling distributions a transition to a ferromagnetic phase may occur. This transition is related to the ferromagnetic susceptibility

$$
\chi_{F M}:=\frac{1}{N} \sum_{i, j} \overline{\left\langle S_{i} S_{j}\right\rangle_{c}}=\frac{1}{N} \sum_{i, j} \lim _{n \rightarrow 0} \frac{1}{n} \sum_{a}\left\langle S_{i}^{a} S_{j}^{a}\right\rangle_{r e p l}=\frac{1}{\Lambda^{(1)}}
$$

and its divergence is hence linked with another eigenvalue of $\mathcal{H}_{P M}$.

\section{Examples}

We now discuss the results for the three particular models defined before. For the SK-model we find from (26)

$$
\Lambda_{S K}^{(k)}=1-2 \delta_{2, k} \beta^{2}
$$

In accordance with the symmetry $P_{S K}(-J)=P_{S K}(J)$ (cf. (2)) only a transition to a spin-glass phase is possible at low temperature. Defining the transition temperature $T_{c}$ by $\Lambda_{S K}^{(2)}=0$ we find $T_{c}=\sqrt{2}$ and

$$
\chi_{S G}=\frac{T^{2}}{T^{2}-T_{c}^{2}}
$$

which coincides with the well-known results [1].

The expression for the eigenvalues of the Hessian for the VB-model

$$
\Lambda_{V B}^{(k)}=1-\kappa \int \mathrm{d} J p(J) \tanh ^{k}(\beta J)+\kappa \delta_{k, 0}
$$

was already derived by Monasson [26]. Since $\tanh (\beta J) \leq 1$ the integrals become smaller with increasing $k$ while the fluctuations corresponding to $k=0$ are suppressed by the constraint (11). For symmetric distributions $p(J)$ the integral for $k=1$ vanishes identically and hence $\Lambda_{V B}^{(2)}$ is the first eigenvalue to become negative. From $\Lambda_{V R}^{(2)}=0$ results the well-known expression [13] for the critical temperature of the spin-glass transition in the VB-model [13, 15, 16]:

$$
1=\kappa \int \mathrm{d} J p(J) \tanh ^{2}\left(\beta_{c} J\right)
$$

For the Lévy glass we have

$$
\Lambda_{\text {Levy }}^{(k)}=1+\int \frac{\mathrm{d} J \mathrm{~d} \tilde{J}}{2 \pi}|\tilde{J}|^{\alpha} \exp (i J \tilde{J}) \tanh ^{k}(\beta J) .
$$

Again $P_{\alpha}(J)=P_{\alpha}(-J)$ ensures that

$$
\Lambda_{L e v y}^{(2)}=1+\int \frac{\mathrm{d} J \mathrm{~d} \tilde{J}}{2 \pi}|\tilde{J}|^{\alpha} \exp (i J \tilde{J}) \tanh ^{2}(\beta J)=1-\frac{\Gamma(\alpha+1)}{\pi} \sin \left(\frac{\alpha \pi}{2}\right) \int \frac{\mathrm{d} J}{|J|^{\alpha+1}} \tanh ^{2}(\beta J)
$$

is the first eigenvalue to become negative. For the critical temperature of this spin-glass transition we hence find

$$
T_{c}^{\alpha}=C(\alpha) \int \frac{\mathrm{d} J}{|J|^{\alpha+1}} \tanh ^{2}(J) \quad \text { with } \quad C(\alpha)=\frac{\Gamma(\alpha+1)}{\pi} \sin \left(\frac{\alpha \pi}{2}\right)
$$


which was previously derived by both the cavity [19] and the replica method [18]. For the SG-susceptibility of this model results

$$
\chi_{S G}=\frac{T^{\alpha}}{T^{\alpha}-T_{c}^{\alpha}}
$$

which is similar to the expression in the SK-model and in fact comprises it for $\alpha=2$.

We therefore reproduce for all three examples the known results about the instability of the paramagnetic phase. However, it remains unclear at this point whether these instabilities can be cured within the replica-symmetric sector or whether replica symmetry breaking is necessary to stabilize the saddle-point. This question can only be addressed by investigating the more complicated situation with a non-trivial form of $c\left(\sum_{a} \sigma_{a}\right)$ to which we turn now.

\section{THE GENERAL CASE}

At low temperatures or $h_{\text {ext }} \neq 0$ the Hessian $\mathcal{H}$ explicitly depends on $\hat{\sigma}_{3}$ and the determination of its eigenvalues becomes much more complicated. The essential steps of the analysis are as follows: We first use the permutation symmetry between replica indices characteristic for any RS solution to block-diagonalize the Hessian according to the irreducible representations of the permutation group. For the remaining diagonalization inside the blocks the limit $n \rightarrow 0$ can be performed and the eigenvalue equations assume the form of integral equations. We then show that the replicon eigenvalue related to the spin-glass susceptibility can only belong to one of the first three blocks. By an expansion around $T_{c}$ we then verify that all eigenvalues of the zeroth and first block which go to zero at $T_{c}$ return to positive values below $T_{c}$. Consequently the replicon eigenvalue must lie in the second block. Finally we identify this eigenvalue and give explicit expressions for the AT-line for the three special models considered.

A major simplification of the general eigenvalue problem of the Hessian is obtained by invoking the symmetry of $\mathcal{H}$ under permutations of the replica indices. Formally this symmetry is expressed by the commutation of the Hessian with a representation $D$ of the permutation group $\Sigma_{n}$. In our case $D$ is defined by

$$
D(\pi)\left|\sigma_{1}, \sigma_{2} \ldots \sigma_{n}\right\rangle=\left|\sigma_{\pi(1)}, \sigma_{\pi(2)} \ldots \sigma_{\pi(n)}\right\rangle \quad\left|\sigma_{a}\right\rangle \in\{|+\rangle,|-\rangle\} \quad \pi \in \Sigma_{n},
$$

which clearly commutes with $\mathcal{H}$, i.e.

$$
D(\pi) \mathcal{H}=\mathcal{H} D(\pi) \quad \forall \pi \in \Sigma_{n} .
$$

The Hessian can therefore be block-diagonalized according to the irreducible representations $D^{(\rho)}$ of $\Sigma_{n}$ that are contained in $D$. As a first step of the reduction of $D$ we note that the subspaces $V^{(\sigma)}$, spanned by vectors $\left|\sigma_{1}, \sigma_{2} \ldots \sigma_{n}\right\rangle$ with a fixed number $\sigma$ of entries $\sigma_{a}$ equal to plus one, are invariant under $D$. The restricted representation $\Delta^{(\sigma)}$ of $D$ on the subspace $V^{(\sigma)} \sigma=0 \ldots n$ can be further decomposed into irreducible representations:

$$
\Delta^{(\sigma)} \simeq D^{(0)} \oplus D^{(1)} \cdots \oplus D^{(\tilde{\sigma})}
$$

with $\tilde{\sigma}=\min \{\sigma, n-\sigma\}$. The irreducibility of the $D^{(\rho)}$ 's is shown in [21]. Each representation $D^{(\rho)}$ gives rise to an eigenvalue $\lambda^{(\rho)}$ of $\mathcal{H}$, with degeneracy

$$
\operatorname{deg}\left(\lambda^{(\rho)}\right)=\operatorname{dim}\left(D^{(\rho)}\right)=\left(\begin{array}{l}
n \\
\rho
\end{array}\right)-\left(\begin{array}{c}
n \\
\rho-1
\end{array}\right) .
$$

The subspaces $V^{(\sigma)}$ hence split into direct sums of irreducible subspaces $V^{(\sigma, \rho)}$ each of which is associated with a representation $D^{(\rho)}$,

$$
V^{(\sigma)} \simeq V^{(\sigma, 0)} \oplus V^{(\sigma, 1)} \cdots \oplus V^{(\sigma, \tilde{\sigma})} .
$$

This decomposition can be accomplished by Young-symmetrizers constructed using the Young tableaus [24]. The procedure shows that the vectors

$$
\| \sigma ; \rho\rangle\rangle:=(|+\rangle|-\rangle)^{\rho}|+\rangle^{\sigma-\rho}|-\rangle^{n-\sigma-\rho} \in V^{(\sigma)}, \quad \rho=0 \ldots \tilde{\sigma},
$$

are mapped onto the irreducible invariant subspace $V^{(\sigma, \rho)}$ by an anti-symmetrization in two successive entries in the first $2 \rho$ arguments, and a complete symmetrization in the last $n-2 \rho$ entries. The vector

$$
\left.\left.|\sigma ; \rho\rangle:=\mathcal{A}_{2 \rho} \mathcal{S}_{n-2 \rho}(\sigma) \| \sigma ; \rho\right\rangle\right\rangle=(|+\rangle|-\rangle-|-\rangle|+\rangle)^{\rho}|\sigma-\rho\rangle_{n-2 \rho}
$$


hence lies in $V^{(\sigma, \rho)}$, where the operators $\mathcal{A}_{2 \rho}$ and $\mathcal{S}_{n-2 \rho}(\sigma)$ denote the anti-symmetrization and the symmetrization operators, respectively and the symmetrized part of the vector $|\sigma ; \rho\rangle$ with $\sigma-\rho$ entries equal to plus one is denoted by

$$
|\sigma-\rho\rangle_{n-2 \rho}=\sum_{\sum \sigma_{a}=2 \sigma-n} \bigotimes_{a=1}^{n-2 \rho}\left|\sigma_{a}\right\rangle
$$

A basis of the subspace $V^{(\sigma, \rho)}$ can be constructed by applying all the $D(\pi)$ on $|\sigma ; \rho\rangle$ and choosing a maximal linearly independent subset. We note that the vectors $\{|\sigma ; \rho\rangle, \sigma=0 \ldots n, \quad \rho=0 \ldots \tilde{\sigma}\}$ are orthogonal, but not normalized. For a fixed $\rho$ the set of the normalized vectors

$$
\left\{\frac{|\sigma ; \rho\rangle}{\sqrt{\langle\sigma ; \rho \mid \sigma ; \rho\rangle}}, \quad \sigma=\rho \ldots n-\rho\right\}
$$

is an orthonormal basis of a subspace $W^{(\rho)}$ of $V$, containing one element from each irreducible subspace $V^{(\sigma, \rho)}, \sigma=\rho \ldots n-\rho$. The matrix $\mathcal{H}_{\mathrm{Sym}}^{(\rho)}$ with matrix elements

$$
\mathcal{H}_{\mathrm{Sym}}^{(\rho)}(\sigma, \tau)=\frac{\langle\sigma ; \rho|\mathcal{H}| \tau ; \rho\rangle}{\sqrt{\langle\sigma ; \rho \mid \sigma ; \rho\rangle\langle\tau ; \rho \mid \tau ; \rho\rangle}} \quad \sigma, \tau=\rho \ldots n-\rho
$$

therefore comprises information from each irreducible subspace $V^{(\sigma, \rho)}, \quad \sigma=\rho \ldots n-\rho$ 22, 23, 25] . Diagonalization of the $n+1-2 \rho$ dimensional matrix $\mathcal{H}_{\mathrm{Sym}}^{(\rho)}$ leads to the eigenvalues

$$
\lambda_{\sigma}^{(\rho)}, \quad \sigma=\rho \ldots n-\rho
$$

each of which is associated with one representation $D^{(\rho)}$ arising in the decomposition of $D$.

Exploiting the symmetry of the problem we have hence reduced the $2^{n}$ dimensional eigenvalue problem to $\left\lfloor\frac{n}{2}\right\rfloor$ eigenvalue equations of dimension $n+1-2 \rho$ which are parametrized by $\rho$. Here $\lfloor x\rfloor$ denotes the largest integer smaller than $x$. As shown in appendix $\mathrm{B}$ the permutation symmetry of $\mathcal{H}$ can be further used to switch from the eigenvalue problem with symmetric matrices $\mathcal{H}_{\text {Sym }}^{(\rho)}$ to non-symmetric matrices with matrix elements $\mathcal{H}^{(\rho)}(\sigma, \tau)=\langle\langle\sigma ; \rho \| \mathcal{H} \mid \tau ; \rho\rangle$. This form allows an elegant continuation $n \rightarrow 0$ which was already used in [22, 23]. We expect that the eigenvalues of the matrices $\mathcal{H}^{(\rho)}$ are generically non-degenerated since the permutation symmetry is already completely accounted for.

Finally the eigenvalues $\lambda^{(\rho)}$ corresponding to the representations $D^{(\rho)}$ have to be determined from the eigenvalue equation:

$$
\lambda^{(\rho)} \chi^{(\rho)}(\tau)=\sum_{\sigma=\rho}^{n-\rho} \mathcal{H}^{(\rho)}(\sigma, \tau) \chi^{(\rho)}(\sigma)
$$

The decomposition $\mathcal{H}=\mathcal{H}_{S}+\mathcal{H}_{E}$ of the Hessian still holds in the new basis. The entropic part $\mathcal{H}_{S}$ is diagonal and depends on the RS solution $c\left(\sum_{a} \sigma_{a}\right)$ of the saddle-point equation only:

$$
\mathcal{H}_{S}^{(\rho)}(\sigma, \tau)=\frac{\delta_{\sigma, \tau}}{c(2 \sigma-n)} .
$$

The energetic part $\mathcal{H}_{E}^{(\rho)}$ depends on the details of the Hamiltonian and will be specified in the treatment of the particular models below. For the following analysis it is convenient to multiply the eigenvalue equations with the inverse of $\mathcal{H}_{S}^{(\rho)}$ and to transform the eigenvectors $\chi^{(\rho)}$ to functions $\phi^{(\rho)}$ according to [22, 23]

$$
\phi^{(\rho)}(h):=\sum_{\sigma=\rho}^{n-\rho} \chi^{(\rho)}(\sigma) \exp (\beta h(2 \sigma-n)) .
$$

The limit $n \rightarrow 0$ may then be performed which transforms the finite dimensional matrix equations into integral equations. In the case of the Lévy glass it also allows the continuation to real temperature. Some intermediate steps of the calculations are given in appendix $\mathrm{C}$.

Eventually we arrive at eigenvalue equations of the form

$$
\lambda^{(\rho)} \int \mathrm{d} \tilde{h} \mathcal{P}(\tilde{h}) \phi^{(\rho)}(\tilde{h}+h)=\phi^{(\rho)}(h)-\int \mathrm{d} \tilde{h} \mathcal{P}(\tilde{h}) \int \mathrm{d} J G_{M}(J)\left(\partial_{h} u\right)^{\rho}(\tilde{h}+h, J) \phi^{(\rho)}(u(\tilde{h}+h, J))
$$


where $u(h, J)=\frac{1}{\beta} \operatorname{atanh}(\tanh (\beta h) \tanh (\beta J))$ and $\mathcal{P}(h)$ denotes as before the replica-symmetric distribution of local fields. This equation is the central result of the present section.

As a first test we reproduce the spectrum of the Hessian obtained in section 【II for $h_{\text {ext }}=0$ and $T \geq T_{c}$. In this case $\mathcal{P}(h)=\delta(h)$ and the eigenvalue equations simplify to

$$
\lambda^{(\rho)} \phi^{(\rho)}(h)=\phi^{(\rho)}(h)-\int \mathrm{d} J G_{M}(J)\left(\partial_{h} u\right)^{\rho}(h, J) \phi^{(\rho)}(u(h, J)) .
$$

Setting $h=0$ and using $u(h=0, J)=0$ as well as $\partial_{h} u(h=0, J)=\tanh (\beta J)$ we get

$$
\lambda^{(\rho)} \phi^{(\rho)}(0)=\phi^{(\rho)}(0)-\int \mathrm{d} J G_{M}(J) \tanh ^{\rho}(\beta J) \phi^{(\rho)}(0) .
$$

If $\phi^{(\rho)}(0) \neq 0$ we hence find $\Lambda^{(\rho)}=\lambda^{(\rho)}$ and therefore reproduce expression (26) for the eigenvalues obtained more directly in section III. If on the other hand $\phi^{(\rho)}(0)=0$, then eq. (51) does not convey any information about $\lambda^{(\rho)}$. However, in this case we find after differentiating (50) with respect to $h$ and setting $h=0$ afterwards

$$
\lambda^{(\rho)}\left(\phi^{(\rho)}\right)^{\prime}(0)=\left(\phi^{(\rho)}\right)^{\prime}(0)-\int \mathrm{d} J G_{M}(J) \tanh ^{(\rho+1)}(\beta J)\left(\phi^{(\rho)}\right)^{\prime}(0) .
$$

If $\left(\phi^{(\rho)}\right)^{\prime}(0) \neq 0$ this returns the expression for $\Lambda^{(\rho+1)}$. If $\left(\phi^{(\rho)}\right)^{\prime}(0)=0$ we turn to the second derivative of (51) and so on. In conclusion we find that for $h_{\text {ext }}=0$ and $T \geq T_{c}$ the sub-block $\mathcal{H}^{(\rho)}$ defined in (44) generates all eigenvalues $\Lambda^{(k)}$ from (26) with $k \geq \rho$. Conversely the eigenvalue $\Lambda^{(2)}$ which signals the spin-glass transition shows up only in blocks $\rho=0,1,2$ implying that in some neighbourhood of $T_{c}$ all eigenvalues $\lambda^{(\rho)}$ with $\rho>2$ are strictly positive. This is also corroborated by a replica representation of the spin-glass susceptibility starting with (27) which shows that the SG susceptibility does not depend on eigenvalues $\lambda^{(\rho)}$ with $\rho>2$. In the following we therefore investigate only the "dangerous" blocks $\rho=0,1,2$.

For $\rho=0$ the constant function $\phi^{(0)}(h)=1$ is an eigenfunction corresponding to the eigenvalue $\lambda^{(0)}=1=\Lambda^{(0)}$. In the high temperature region this eigenvalue was irrelevant for the stability due to the constraint (11). We assume that the same holds true in the spin-glass phase as well. For the first derivative of (49) we find for $(\rho=0)$

$$
\lambda^{(0)} \int \mathrm{d} \tilde{h} \mathcal{P}(\tilde{h})\left(\phi^{(0)}\right)^{\prime}(\tilde{h}+h)=\left(\phi^{(0)}\right)^{\prime}(h)-\int \mathrm{d} \tilde{h} \mathcal{P}(\tilde{h}) \int \mathrm{d} J G_{M}(J) \partial_{h} u(\tilde{h}+h, J)\left(\phi^{(0)}\right)^{\prime}(u(\tilde{h}+h, J)) .
$$

Hence either $\left(\phi^{(0)}\right)^{\prime} \equiv 0$ or $\left(\phi^{(0)}\right)^{\prime}$ is an eigenfunction of (49) for $\rho=1$. Conversely if $\phi^{(1)}$ is an eigenfunction of (49) for $\rho=1$ its primitive $\Phi^{(1)}$ satisfying $\left(\Phi^{(1)}\right)^{\prime}=\phi^{(1)}$ fulfills the equation

$$
\partial_{h}\left\{\lambda^{(1)} \int \mathrm{d} \tilde{h} \mathcal{P}(\tilde{h}) \Phi^{(1)}(\tilde{h}+h)\right\}=\partial_{h}\left\{\Phi^{(1)}(h)-\int \mathrm{d} \tilde{h} \mathcal{P}(\tilde{h}) \int \mathrm{d} J G_{M}(J) \Phi^{(1)}(u(\tilde{h}+h, J))\right\} .
$$

Integration of this equation yields an eigenfunction $\Phi^{(1)}$ of (49) for $\rho=0$ since the integration constant may be absorbed in the choice of $\Phi^{(1)}$. Consequently the block with $\rho=0$ contains the same eigenvalues as the block corresponding to $\rho=1$ and in addition one eigenvalue corresponding to a constant eigenfunction which we believe to be irrelevant due to the constraint (11). This degeneracy between the $\rho=0$ and the $\rho=1$ block is similar to the well-known degeneracy of the longitudinal and first transversal eigenvalue in the stability analysis of the SK model [5].

We now show that the eigenvalues $\lambda^{(0)}$ and $\lambda^{(1)}$ which are degenerate with $\Lambda^{(2)}$ in the high temperature phase return to positive values below $T_{c}$. In view of the equivalence between the eigenvalues from the zeroth and first block it is sufficient to show this for $\lambda^{(1)}$. We study the eigenvalue equation perturbatively to leading order in the reduced temperature $\tau=1-T / T_{c}$ at zero external field. To this end we expand the derivative of the $\rho=1$ eigenvalue equation with respect to $h$ at $h=0$ up to order $h^{2}$ [27]. The integral equation acquires the form of a 2-dimensional matrix eigenvalue problem. To leading order in $\tau$ we find

$$
\lambda_{S K}^{(1)}=2 \tau, \quad \lambda_{V B}^{(1)}=t_{2}^{\prime} \tau, \quad \lambda_{L}^{(1)}=\alpha \tau,
$$

where $t_{2}^{\prime}$ is defined as

$$
t_{2}^{\prime}:=\left.\frac{\mathrm{d}}{\mathrm{d} \tau} \kappa \int \mathrm{d} J p(J) \tanh ^{2}\left(\frac{\beta_{c} J}{1-\tau}\right)\right|_{\tau=0}>0 .
$$

In all three cases the eigenvalue hence returns to positive values. The instability of the paramagnetic saddle-point due to unstable directions from the zeroth and first block are therefore cured by the replica symmetric low-temperature solution. The "dangerous" direction related to the replicon eigenvalue is contained in the $\rho=2$ sector. Its detailed discussion requires a specification of the Hamiltonian which we therefore perform separately for the three case of interest. 
The SK Model

Using $G_{S K}(J)=\delta^{\prime \prime}(J)$ in (49) for $\rho=2$ we find:

$$
\lambda^{(2)} \int \mathrm{d} \tilde{h} \mathcal{P}(\tilde{h}) \phi^{(2)}(\tilde{h}+h)=\phi^{(2)}(h)-2 \beta^{2} \phi^{(2)}(0) \int \mathrm{d} \tilde{h} \mathcal{P}(\tilde{h}) \frac{1}{\cosh ^{4}(\beta(h+\tilde{h}))} .
$$

At zero external field close to the transition temperature this equation can also be studied perturbatively in the reduced temperature $\tau$. An expansion of the last equation at $h=0$ up to order $h^{4}$ turns the integral equation to a three dimensional eigenvalue problem. One eigenvalue becomes negative:

$$
\lambda^{(2)}=-\frac{4}{3} \tau^{2}+\mathcal{O}\left(\tau^{3}\right),
$$

indicating the well-known instability of the RS solution.

In the presence of an external field we identify $\lambda^{(2)}=0$ with the instability-line, which starts at $T_{c}$ for $h_{\text {ext }}=0$. The replica symmetric distribution of local fields is explicitly known for the SK model:

$$
\mathcal{P}(h)=\frac{1}{\sqrt{4 \pi q}} \exp \left(-\frac{\left(h-h_{\mathrm{ext}}\right)^{2}}{4 q}\right)
$$

where in the spin-glass phase $q$ is the non-zero solution of $q=\int \mathrm{d} h \mathcal{P}(h) \tanh ^{2}(\beta h)$. Setting $\lambda^{(2)}=0$ and $h=0$ in (57) we arrive at

$$
1=2 \beta^{2} \int \frac{\mathrm{d} x}{\sqrt{2 \pi}} \frac{\exp \left(-\frac{x^{2}}{2}\right)}{\cosh ^{4}\left(\beta\left(\sqrt{2 q} x+h_{\mathrm{ext}}\right)\right)}
$$

which reproduces the famous AT-line for the SK-model in the $h_{\text {ext }}-T$-plane [5].

The VB Model

In the case of the VB model the eigenvalue equation for $\rho=2$ reads:

$$
\lambda^{(2)} \int \mathrm{d} \tilde{h} \mathcal{P}(\tilde{h}) \phi^{(2)}(\tilde{h}+h)=\phi^{(2)}(h)-\kappa \int \mathrm{d} \tilde{h} \mathcal{P}(\tilde{h}) \int \mathrm{d} J p(J)\left(\partial_{h} u\right)^{2}(\tilde{h}+h, J) \phi^{(2)}(u(\tilde{h}+h, J)) .
$$

At zero external field an expansion of the eigenvalue equation (61) can be invoked leading to the eigenvalue

$$
\lambda^{(2)}=-\frac{1}{3} \frac{1+2 t_{4}}{1-t_{4}} t_{2}^{\prime 2} \tau^{2}+\mathcal{O}\left(\tau^{3}\right)
$$

with

$$
t_{4}:=\kappa \int \mathrm{d} J p(J) \tanh ^{4}\left(\beta_{c} J\right)<\kappa \int \mathrm{d} J p(J) \tanh ^{2}\left(\beta_{c} J\right)=1,
$$

and $t_{2}^{\prime}$ defined in eq. (56). Due to the inequality in the last line $\lambda^{(2)}$ is negative below $T_{c}$ indicating the instability of the replica symmetric solution for this model.

Within the cavity approach the AT-line is described by [28]

$$
\mu \phi(h)=\kappa \int \mathrm{d} \tilde{h} \mathcal{P}(\tilde{h}) \int \mathrm{d} J p(J)\left(\partial_{h} u\right)^{2}(\tilde{h}+h, J) \phi(u(\tilde{h}+h, J)),
$$

where the RS phase becomes unstable when the largest eigenvalue $\mu$ exceeds the value 1 [29]. Since (61) for $\lambda^{(2)}=0$ and (63) for $\mu=1$ coincide we have reproduced the stability criterion from the cavity method within the replica approach also for non-zero external field.

The largest eigenvalue of eq. (63) can be determined numerically by simple iteration. Unfortunately we do not know about a similar straightforward method to determine the smallest eigenvalue $\lambda^{(2)}$ of eq. (61). 
The eigenvalue equation for the Lévy SG is similar to the one for the VB-model:

$$
\lambda^{(2)} \int \mathrm{d} \tilde{h} \mathcal{P}(\tilde{h}) \phi^{(2)}(\tilde{h}+h)=\phi^{(2)}(h)-C(\alpha) \int \mathrm{d} \tilde{h} \mathcal{P}(\tilde{h}) \int \frac{\mathrm{d} J}{|J|^{\alpha+1}}\left(\partial_{h} u\right)^{2}(\tilde{h}+h, J) \phi^{(2)}(u(\tilde{h}+h, J)),
$$

where $C(\alpha)$ was defined in (35). The expansion of the eigenvalue equation (64) to the leading order in the reduced temperature amounts to

$$
\lambda^{(2)}=-\frac{\alpha^{2}}{3} \frac{1+2 t_{4, \alpha}}{1-t_{4, \alpha}} \tau^{2}+\mathcal{O}\left(\tau^{3}\right),
$$

with

$$
t_{4, \alpha}:=C(\alpha) \int \frac{\mathrm{d} J}{|J|^{\alpha+1}} \tanh ^{4}\left(\beta_{c} J\right)<C(\alpha) \int \frac{\mathrm{d} J}{|J|^{\alpha+1}} \tanh ^{2}\left(\beta_{c} J\right)=1 .
$$

This proves that $\lambda^{(2)}$ is indeed negative below $T_{c}$ and the replica symmetric phase is unstable below $T_{c}$.

The stability analysis for the Lévy SG performed in [28] using the cavity method gave rise to the equation

$$
\mu \phi(h)=C(\alpha) \int \mathrm{d} \tilde{h} \mathcal{P}(\tilde{h}) \int \frac{\mathrm{d} J}{|J|^{\alpha+1}}\left(\partial_{h} u\right)^{2}(\tilde{h}+h, J) \phi(u(\tilde{h}+h, J)),
$$

where the instability of the RS solution was again signaled by $\mu>1$ [30]. In the presence of an external field we therefore find equivalence between the results obtained using the cavity and the replica method.

In the large connectivity limit of the VB model and in the SK-limit $(\alpha \rightarrow 2)$ of the Lévy SG all our results are consistent with each other. In view of (12) we have $\lim _{\alpha \rightarrow 2} G_{L}(J)=\delta^{\prime \prime}(J)=G_{S K}(J)$. To obtain the large connectivity limit of the AT-line for the VB model we use (5) and (6) respectively in (61). In the limit $N \rightarrow \infty$ the eigenvalue equation then acquires the desired form up to a constant depending on the details of the distribution $p(J)$ which can be absorbed in the energy scale.

To see the equivalence for the eigenvalues close to the transition temperature we use

$$
t_{2}^{\prime} \rightarrow \alpha \rightarrow 2 \quad \text { and } \quad t_{4} \rightarrow t_{4, \alpha} \rightarrow t_{4,2}=\int \mathrm{d} J \delta^{\prime \prime}(J) \tanh ^{4}\left(\beta_{c} J\right)=0
$$

where the first arrow corresponds to the Lévy limit of the VB model, and the second one to the SK-limit of the Lévy SG. If the second moment of the distribution $p(J)$ exists, one obtains using (5) directly $t_{2}^{\prime} \rightarrow 2$ and $t_{4} \rightarrow 0$.

\section{SUMMARY}

In the present paper we derived within the replica formalism expressions for the AT-line of general mean-field spin-glasses including strongly diluted and Lévy spin glasses. Due to the non-Gaussian character of the local field distribution in these models an infinite number of order parameters is needed already at the replica symmetric level. Following the approach of Monasson the fluctuations around the replica symmetric saddle-point are described by an $2^{n} \times 2^{n}$ Hessian matrix.

At high temperatures and in zero external field the distribution of local fields is a delta-function and the determination of the eigenvalues of this Hessian is relatively straightforward. We find that all eigenvalues are positive at sufficiently high temperature and that some of them tend to zero at the critical temperature, $T_{c}$, which signals the transition to the glass phase.

Below the critical temperature the RS order parameter develops a non-trivial structure and the determination of the spectrum of the Hessian becomes rather involved. However, using the symmetry of the saddle-point under permutations of the replicas the Hessian can be block-diagonalized and the sub-blocks relevant for the stability of RS can be identified. Performing the $n \rightarrow 0$ limit in these blocks turns the finite dimensional eigenvalue equations into integral equations from which general expressions for the AT-lines may be derived.

We show the validity of our approach by applying it to three representative model systems: the SK-model as the standard model with Gaussian field distribution, the VB-model as the standard model for diluted spin-glasses for which higher moments of the field distribution are essential, and the Lévy spin glass as standard model for spin-glasses with diverging second moment of the coupling distribution.

We believe that with our stability analysis the replica-symmetric theory of general spin-glass models is now complete. 


\section{Acknowledgments}

We would like to thank Marc Mézard, Remi Monasson and Martin Weigt for interesting discussions. Financial support from the Deutsche Forschungsgemeinschaft under EN 278/7 is gratefully acknowledged.

\section{Appendix A: The spectrum of $\mathcal{H}^{(\rho)}$}

First of all we show that the eigenvalues of $\mathcal{H}_{\mathrm{Sym}}^{(\rho)}$ and $\mathcal{H}^{(\rho)}$ coincide. To this end we note that the symmetrization operators $\mathcal{S}_{n-2 \rho}(\sigma)$ and $\mathcal{A}_{2 \rho}$ can be represented as appropriate combinations of $D(\pi), \pi \in \Sigma_{n}$. The operator $\mathcal{S}_{n-2 \rho}(\sigma)$, as a sum of $\left(\begin{array}{c}n-2 \rho \\ \sigma-\rho\end{array}\right)$ permutations, and $\mathcal{A}_{2 \rho}$ as a product

$$
\mathcal{A}_{2 \rho}=\prod_{a=1}^{\rho}[D(e)-D((2 a, 2 a-1))],
$$

where $e$ denotes the identity of the group and $(2 a, 2 a-1)$ the transposition of the elements $2 a$ and $2 a-1$. Being elements of the group algebra all operators $\mathcal{S}$ and $\mathcal{A}$ commute with $\mathcal{H}$. Therefore

$$
\langle\sigma ; \rho|\mathcal{H}| \tau ; \rho\rangle=\left\langle\left\langle\sigma ; \rho \| \mathcal{H} \mathcal{A}_{2 \rho} \mathcal{S}_{n-2 \rho}(\sigma) \mid \tau ; \rho\right\rangle\right.
$$

The vector $|\tau ; \rho\rangle$ is symmetric in the last $n-2 \rho$ entries. The action of any $D(\pi)$ acting only on these last entries is hence trivial and we find

$$
\mathcal{S}_{n-2 \rho}(\sigma)|\tau ; \rho\rangle=\left(\begin{array}{c}
n-2 \rho \\
\sigma-\rho
\end{array}\right)|\tau ; \rho\rangle,
$$

since $\mathcal{S}_{n-2 \rho}(\sigma)$ is a sum of $\left(\begin{array}{c}n-2 \rho \\ \sigma-\rho\end{array}\right)$ permutations. From $[D(e)-D((1,2))](|+\rangle|-\rangle-|-\rangle|+\rangle)=2(|+\rangle|-\rangle-|-\rangle|+\rangle)$, one similarly derives $\mathcal{A}_{2 \rho}|\tau ; \rho\rangle=2^{\rho}|\tau ; \rho\rangle$. The action of $\mathcal{A}_{2 \rho} \mathcal{S}_{n-2 \rho}(\sigma)$ reproduces $|\tau ; \rho\rangle$ up to the constant $2^{\rho}\left(\begin{array}{c}n-2 \rho \\ \sigma-\rho\end{array}\right)$ which is the squared norm of $|\sigma ; \rho\rangle$. One hence has the following relation between the matrix elements of $\mathcal{H}$ and $\mathcal{H}^{(\rho)}$ : $\langle\sigma ; \rho|\mathcal{H}| \tau ; \rho\rangle=\langle\sigma ; \rho \mid \sigma ; \rho\rangle \mathcal{H}^{(\rho)}(\sigma, \tau)$. Using this relation in the eigenvalue equation for $\mathcal{H}_{\text {Sym }}^{(\rho)}$ :

$$
\begin{aligned}
\lambda^{(\rho)} \tilde{\chi}^{(\rho)}(\tau) & =\sum_{\sigma=\rho}^{n-\rho} \mathcal{H}_{\mathrm{Sym}}^{(\rho)}(\sigma, \tau) \tilde{\chi}^{(\rho)}(\sigma)=\sum_{\sigma=\rho}^{n-\rho} \sqrt{\frac{\langle\sigma ; \rho \mid \sigma ; \rho\rangle}{\langle\tau ; \rho \mid \tau ; \rho\rangle}} \mathcal{H}^{(\rho)}(\sigma, \tau) \tilde{\chi}^{(\rho)}(\sigma) \\
\Leftrightarrow \quad \lambda^{(\rho)} \chi^{(\rho)}(\tau) & =\sum_{\sigma=\rho}^{n-\rho} \mathcal{H}^{(\rho)}(\sigma, \tau) \chi^{(\rho)}(\tau), \quad \text { with } \quad \chi^{(\rho)}(\sigma)=\sqrt{\langle\sigma ; \rho \mid \sigma ; \rho\rangle} \tilde{\chi}^{(\rho)}(\sigma)
\end{aligned}
$$

we see that the eigenvalues of the matrices coincide.

\section{Appendix B: The limit $n \rightarrow 0$}

To perform the limit $n \rightarrow 0$ we switch to the characteristic functions defined in (48). Transforming the whole eigenvalue equation (46) one has to calculate the quantity

$$
\sum_{\tau=\rho}^{n-\rho} \mathcal{H}^{(\rho)}(\sigma, \tau) \exp (\beta h(2 \tau-n))
$$

which can be performed without the explicit evaluation of the matrix elements $\mathcal{H}^{(\rho)}(\sigma, \tau)$. As we will see the transformed quantities will allow for a continuation to real $n$. The $n \rightarrow 0$ limit then turns the finite dimensional eigenvalue equation into the integral equation (49).

In section III the entropic and the energetic parts of the Hessian, $\mathcal{H}_{S}$ and $\mathcal{H}_{E}$, were decomposed into a tensor product of identical $2 \times 2$ matrices. Therefore we first calculate the transformation (B1) for a tensor product of $\mathrm{n}$ 
$2 \times 2$ matrices $\mathbf{g}$ and use the general result for the two parts of the Hessian.

$$
\begin{aligned}
& \sum_{\tau=\rho}^{n-\rho}\left\langle\left\langle\sigma ; \rho \| \bigotimes_{a=1}^{n} \mathbf{g} \mid \tau ; \rho\right\rangle \exp (\beta h(2 \tau-n))\right. \\
& =\sum_{\tau=\rho}^{n-\rho}\left(\left\langle+|\langle-|)^{\rho}(\langle+|)^{\sigma-\rho}(\langle-|)^{n-\sigma-\rho} \bigotimes_{a=1}^{n} \mathbf{g}(|+\rangle|-\rangle-|-\rangle|+\rangle)^{\rho} \mid \tau-\rho\right\rangle_{n-2 \rho} \exp (\beta h(2 \tau-n))\right. \\
& =\left(\left\langle+\left|\left\langle-|\mathbf{g} \otimes \mathbf{g}(|+\rangle|-\rangle-|-\rangle|+\rangle))^{\rho} \sum_{\tau=\rho}^{n-\rho}(\langle+|)^{\sigma-\rho}(\langle-|)^{n-\sigma-\rho} \bigotimes_{a=1}^{n-2 \rho} \mathbf{g}\left[\sum_{\sum \tau_{a}=2 \tau-n} \bigotimes_{a=1}^{n-2 \rho}\left|\tau_{a}\right\rangle\right] \exp \left(\beta h \sum_{a=\rho}^{n-\rho} \tau_{a}\right)\right.\right.\right.\right. \\
& =(\langle+|\mathbf{g}|+\rangle\langle-|\mathbf{g}|-\rangle-\langle+|\mathbf{g}|-\rangle\langle-|\mathbf{g}|+\rangle)^{\rho} \sum_{\left\{\tau_{a}= \pm 1\right\}}\left[\prod_{b=1}^{\sigma-\rho}\left\langle+|\mathbf{g}| \tau_{b}\right\rangle e^{\beta h \tau_{b}} \prod_{c=1}^{n-\sigma-\rho}\left\langle-|\mathbf{g}| \tau_{c}\right\rangle e^{\beta h \tau_{c}}\right] \\
& =\left(\mathbf{g}_{++} \mathbf{g}_{--}-\mathbf{g}_{+-} \mathbf{g}_{-+}\right)^{\rho}\left(\sum_{\tau_{b}= \pm}\left\langle+|\mathbf{g}| \tau_{b}\right\rangle e^{\beta h \tau_{b}}\right)^{\sigma-\rho}\left(\sum_{\tau_{c}= \pm}\left\langle-|\mathbf{g}| \tau_{c}\right\rangle e^{\beta h \tau_{c}}\right)^{n-\sigma-\rho} \\
& =\left(\mathbf{g}_{++} \mathbf{g}_{--}-\mathbf{g}_{+-} \mathbf{g}_{-+}\right)^{\rho}\left(\mathbf{g}_{++} e^{\beta h}+\mathbf{g}_{+-} e^{-\beta h}\right)^{\sigma-\rho}\left(\mathbf{g}_{+-} e^{\beta h}+\mathbf{g}_{--} e^{-\beta h}\right)^{n-\sigma-\rho}
\end{aligned}
$$

where $\mathbf{g}_{\sigma \tau}:=\langle\sigma|\mathbf{g}| \tau\rangle, \sigma, \tau \in \pm$ denote the matrix elements of $\mathbf{g}$. In the second line we have used the definitions of $\langle\sigma \sigma \rho \|$ and $\mid \tau ; \rho\rangle$ and then decomposed the symmetric part of $|\tau ; \rho\rangle$ as in (42), using $2 \tau-n=\sum_{a} \tau_{a}$.

The entropic contribution (17) can be written as

$$
\mathcal{H}_{S}=\int \frac{\mathrm{d} r \mathrm{~d} r^{\prime}}{2 \pi} \frac{e^{i r r^{\prime}}}{c(r)} \bigotimes_{a=1}^{n} \exp \left(-i r^{\prime} \hat{\sigma}_{3}\right)=\int \frac{\mathrm{d} r \mathrm{~d} r^{\prime}}{2 \pi} \frac{e^{i r r^{\prime}}}{c(r)} \bigotimes_{a=1}^{n} \mathbf{g}^{S}
$$

The entropic matrix $\mathbf{g}^{S}$ is diagonal: $\mathbf{g}_{++}^{S}=e^{-i r^{\prime}}, \quad \mathbf{g}_{--}^{S}=e^{+i r^{\prime}}$, and using (B2) we find

$$
\begin{aligned}
\sum_{\tau=\rho}^{n-\rho} & \mathcal{H}_{S}^{(\rho)}(\sigma, \tau) \exp (\beta h(2 \tau-n))=\int \frac{\mathrm{d} r \mathrm{~d} r^{\prime}}{2 \pi} \frac{e^{i r r^{\prime}}}{c(r)} \sum_{\tau=\rho}^{n-\rho}\left\langle\sigma ; \rho \| \bigotimes_{a=1}^{n} \mathbf{g}^{S}\left(r^{\prime}\right) \mid \tau ; \rho\right\rangle \exp (\beta h(2 \tau-n)) \\
& =\int \frac{\mathrm{d} r \mathrm{~d} r^{\prime}}{2 \pi} \frac{e^{i r r^{\prime}}}{c(r)}\left(\mathbf{g}_{++}^{S} e^{\beta h}\right)^{\sigma-\rho}\left(\mathbf{g}_{--}^{S} e^{-\beta h}\right)^{n-\sigma-\rho}=\int \mathrm{d} r \frac{1}{c(r)} \delta(r-(2 \sigma-n)) \exp (\beta h(2 \sigma-n)) \\
& =\sum_{\tau=\rho}^{n-\rho} \frac{\delta_{\sigma, \tau}}{c(2 \sigma-n)} \exp (\beta h(2 \tau-n)) .
\end{aligned}
$$

The expression in the last line leads to eq. (47). Due to the structure of the RS solution (14) it is convenient to determine the inverse of $\mathcal{H}_{S}^{(\rho)}$ which amounts to :

$$
\left(\mathcal{H}_{S}^{(\rho)}\right)^{-1}(\sigma, \tau)=\delta_{\sigma, \tau} c(2 \sigma-n)=\delta_{\sigma, \tau} \int \mathrm{d} \tilde{h} \mathcal{P}(\tilde{h}) \frac{\exp (\beta \tilde{h}(2 \tau-n))}{(2 \cosh (\beta \tilde{h}))^{n}}
$$

The energetic contribution (16) reads

$$
\mathcal{H}_{E}=-\int \mathrm{d} J G_{M}(J) \bigotimes_{a=1}^{n}\left[e^{\beta J} \hat{\sigma}_{0}+e^{-\beta J} \hat{\sigma}_{1}\right]=-\int \mathrm{d} J G_{M}(J) \bigotimes_{a=1}^{n} \mathrm{~g}^{E}(J),
$$

with diagonal elements $\mathbf{g}_{\sigma \sigma}^{E}(J)=e^{\beta J}$, and off-diagonal terms $\mathbf{g}_{\sigma-\sigma}^{E}=e^{-\beta J}, \sigma= \pm 1$. Using (B2) we get

$$
\begin{aligned}
-\sum_{\tau=\rho}^{n-\rho} \mathcal{H}_{E}^{(\rho)}(\sigma, \tau) \exp (\beta h(2 \tau-n))=\int \mathrm{d} J G_{M}(J) \sum_{\tau=\rho}^{n-\rho}\left\langle\left\langle\sigma ; \rho \| \bigotimes_{a=1}^{n} \mathbf{g}^{E}(J) \mid \tau ; \rho\right\rangle \exp (\beta h(2 \tau-n))\right. \\
\quad=\int \mathrm{d} J G_{M}(J)\left(e^{2 \beta J}-e^{-2 \beta J}\right)^{\rho}\left(e^{\beta(J+h)}+e^{-\beta(J+h)}\right)^{\sigma-\rho}\left(e^{\beta(-J+h)}+e^{-\beta(-J+h)}\right)^{n-\rho-\sigma} \\
=\int \mathrm{d} J G_{M}(J)\left(\partial_{h} u(h, J)\right)^{\rho} w_{n}(h, J) \exp (\beta(2 \sigma-n) u(h, J))
\end{aligned}
$$


with

$$
w_{n}(h, J)=(2 \cosh \beta(J+h) 2 \cosh \beta(J-h))^{\frac{n}{2}}
$$

and

$$
\partial_{h} u(h, J)==\frac{1}{\cosh ^{2}(\beta h)} \frac{\tanh (\beta J)}{1-\tanh ^{2}(\beta J) \tanh ^{2}(\beta h)} .
$$

\section{Appendix C: The eigenvalue equation}

We start from the eigenvalue equation (46) for the matrices $\mathcal{H}^{(\rho)}$. Each of the eigenvalues corresponds to one of the $n+1-2 \rho$ representations $D^{(\rho)}$ which arise in the decomposition (37). Splitting the eigenvalue equation into energetic and entropic part leads to

$$
\lambda^{(\rho)} \chi^{(\rho)}(\tau)=\sum_{\sigma=\rho}^{n-\rho} \mathcal{H}_{S}^{(\rho)}(\sigma, \tau) \chi^{(\rho)}(\tau)+\sum_{\sigma=\rho}^{n-\rho} \mathcal{H}_{E}^{(\rho)}(\sigma, \tau) \chi^{(\rho)}(\sigma)
$$

which is equivalent to

$$
\lambda^{(\rho)} \int \frac{\mathrm{d} \tilde{h} \mathcal{P}(\tilde{h})}{(2 \cosh (\beta \tilde{h}))^{n}} \chi^{(\rho)}(\tau) \exp (\beta \tilde{h}(2 \tau-n))=\chi^{(\rho)}(\tau)+\int \frac{\mathrm{d} \tilde{h} \mathcal{P}(\tilde{h})}{(2 \cosh (\beta \tilde{h}))^{n}} \sum_{\sigma=\rho}^{n-\rho} \mathcal{H}_{E}^{(\rho)}(\sigma, \tau) \exp (\beta \tilde{h}(2 \tau-n)) \chi^{(\rho)}(\sigma) .
$$

Here we have used the explicit form of the inverse of $\mathcal{H}_{S}^{(\rho)}$ given in (B4). We now perform the transformations explained above which allows to perform the limit $n \rightarrow 0$. The transformation of the l.h.s. of the last equation reads

$$
\lambda^{(\rho)} \int \mathrm{d} \tilde{h} \mathcal{P}(\tilde{h}) \frac{1}{(2 \cosh (\beta \tilde{h}))^{n}} \sum_{\tau=\rho}^{n-\rho} \exp (\beta(\tilde{h}+h)(2 \tau-n)) \chi^{(\rho)}(\tau)=\lambda^{(\rho)} \int \mathrm{d} \tilde{h} \frac{1}{(2 \cosh (\beta \tilde{h}))^{n}} \mathcal{P}(\tilde{h}) \phi^{(\rho)}(\tilde{h}+h)
$$

whereas the r.h.s amounts to :

$$
\begin{aligned}
& \sum_{\tau=\rho}^{n-\rho} \chi^{(\rho)}(\tau) \exp (\beta h(2 \tau-n))+\int \mathrm{d} \tilde{h} \mathcal{P}(\tilde{h}) \frac{1}{(2 \cosh (\beta \tilde{h}))^{n}} \sum_{\tau, \sigma=\rho}^{n-\rho} \mathcal{H}_{E}^{(\rho)}(\sigma, \tau) \exp (\beta(\tilde{h}+h)(2 \tau-n)) \chi^{(\rho)}(\sigma) \\
& =\phi^{(\rho)}(h)+\int \mathrm{d} \tilde{h} \mathcal{P}(\tilde{h}) \frac{1}{(2 \cosh (\beta \tilde{h}))^{n}} \sum_{\sigma=\rho}^{n-\rho}\left(\sum_{\tau=\rho}^{n-\rho} \mathcal{H}_{E}^{(\rho)}(\sigma, \tau) \exp (\beta(\tilde{h}+h)(2 \tau-n))\right) \chi^{(\rho)}(\sigma) \\
& =\phi^{(\rho)}(h)-\int \mathrm{d} \tilde{h} \mathcal{P}(\tilde{h}) \frac{1}{(2 \cosh (\beta \tilde{h}))^{n}} \int \mathrm{d} J G_{M}(J)\left(\partial_{h} u(\tilde{h}+h, J)\right)^{\rho} w_{n}(\tilde{h}+h, J) \phi^{(\rho)}(u(\tilde{h}+h, J)),
\end{aligned}
$$

where we have used (B6). The limit $n \rightarrow 0$ may now be performed which yields our central result (49).

[1] Binder K. and Young. A. P., Rev. Mod. Phys. 58, 801 (1986)

[2] Mezard M., Parisi G., and Virasoro M. A., Spin-glass Theory and Beyond (World Scientific, Singapore, 1987)

[3] Engel A. and Van den Broeck C., Statistical Mechanics of Learning, (Cambrigde University Press, Cambrigde, 2001)

[4] Hartmann A. K. and Weigt M., Phase Transitions in Combinatorial Optimization Problems (Wiley VCH, Weinheim, 2005)

[5] de Almeida J. R. L. and Thouless D. J., J. Phys. A 11, 983 (1978)

[6] Edwards S. F. and Anderson P. W., J. Phys. F5, 965 (1975)

[7] Parisi G., in Recent Advances in Field Theory and Statistical Mechanics, Proceedings Les Houches Session XXXIX J.-B. Zuber and R. Stora (eds.), (Elsevier Science Publishers, Amsterdam, 1984)

[8] Mézard M., Parisi G., Virasoro M. A., Europhys. Lett. 1, 77 (1986)

[9] Sherrington D. and Kirkpatrick S., Phys. Rev. Lett. 35, 1972 (1975)

[10] Pytte E. and Rudnick J., Phys. Rev. 19, 3603 (1979)

[11] Bray A. J. and Moore, M. A., J. Phys. C12, 79 (1979)

[12] Monasson R., Zecchina R., Kirkpatrick S., Selman B., and Troyanski L., Nature 400, 133 (1999)

[13] Viana L. and Bray A. J., J. Phys. C18, 3037 (1985) 
[14] Jörg T., Katzgraber H. G., and Krzakala F., Phys. Rev. Lett. 100, 197202 (2008)

[15] Kanter I. and Sompolinski H., Phys. Rev. Lett. 58, 164 (1987)

[16] Mezard M. and Parisi G., Europhys. Lett. 3, 1067 (1987)

[17] Monasson R., J. Phys. A31, 513 (1998)

[18] Janzen K., Hartmann A. K., and Engel A., J. Stat. Mech., P04006 (2008)

[19] Cizeau P. and Bouchaud J.-P., J. Phys. A26, L187 (1993)

[20] Cizeau P., Evenements rares et systemes desordonnes, PhD-thesis, Paris, 1994

[21] Wigner E. Gruppentheorie und ihre Anwendung auf die Quantenmechanik der Atomspektren (F. Vieweg, Braunschweig 1931)

[22] Weigt M. and Monasson R., Europhys. Lett. 36, 209 (1996)

[23] Weigt M., Replikatheorie ungeordneter Systeme: Neuronale Netze und niedrigdimensionale Systeme, PhD-thesis, Magdeburg, Germany, 1998

[24] Weyl H., The classical groups (Princeton university press, 1939)

[25] Pendry J.B., J. Phys. C 15, (1982) 4821

[26] Monasson R., private communication

[27] A similar expansion of an eigenvalue problem was recently used in [28].

[28] Janzen K., Engel A., and Mézard M. arXiv: 1006.2927, accepted for publication in Phys. Rev. E

[29] To obtain this expression the substitution $g_{i} \mapsto g_{i}-h_{i}$ has to be performed in eq. (39) of [28].

[30] The factor $C(\alpha)$ accounts for a slightly different normalization of the Lévy distribution used in [28]. 